\title{
The Problem of Ontology for Spontaneous Collapse Theories
}

\author{
by Bradley Monton \\ Department of Philosophy, University of Kentucky \\ bmonton@uky.edu
}

September 26, 2003 


\title{
The Problem of Ontology for Spontaneous Collapse Theories
}

\begin{abstract}
The question of how to interpret spontaneous collapse theories of quantum mechanics is an open one. One issue involves what link one should use to go from wave function talk to talk of ordinary macroscopic objects. Another issue involves whether that link should be taken ontologically seriously. In this paper, I ague that the link should be taken ontologically seriously; I argue against an ontology consisting solely of the wave function. I then consider three possible links: the fuzzy link, the accessible mass density link, and the mass density simpliciter link. I show that the first two links have serious anomalies which render them unacceptable. I show that the mass density simpliciter link, in contrast, is viable.
\end{abstract}

Keywords: spontaneous localization theories, dynamical reduction theories, GRW theory, wave function ontology, fuzzy link, mass density link 


\section{Introduction}

Spontaneous collapse theories of quantum mechanics (such as the GRW theory of Ghirardi, Rimini, and Weber (1986)) have great philosophical interest. They provide a possible way of solving the measurement problem by changing the dynamics of the theory, and they make wave function collapse philosophically respectable by having it occur independent of observers. Arguably, they can be used to solve fundamental problems in thermodynamics, explaining why entropy generally increases (Albert 1994, 2000). But before philosophers celebrate these virtues of spontaneous collapse theories, an important question should be asked: are these theories viable?

There has recently been an extensive philosophical debate regarding the viability of spontaneous collapse theories. The debate started with Peter Lewis (1997) arguing that spontaneous collapse theories face what has come to be called the counting anomaly: arithmetic applies to ordinary macroscopic objects only as an approximation. One version of the counting anomaly runs as follows. Consider a marble in a box. Because wave functions have tails, the marble will not strictly be in the eigenstate in, of being in the box, but instead will be in the state

$$
|\psi,=a| \text { in, }+b \mid \text { out, },
$$

where $|a| »|b|, b \ldots 0$, and $|a|^{2}+|b|^{2}=1$. According to the eigenstate-eigenvalue link, a marble in state $\mid \psi$, is neither in nor out of the box, but instead is spread throughout an unbounded region of space. It is standardly thought that this is unacceptable, and that the eigenstate-eigenvalue link must be rejected in favor of some interpretative rule which entails that the marble in state $\mid \psi$, is in the box.

Now consider a collection of $n$ marbles in a box, each in state $\mid \psi,$. By the above argument, 
each individual marble is in the box. But the probability of finding all the marbles in the box is $|a|^{2 n}$, which approaches 0 as $n$ gets large. Thus it seems that not all the marbles are in the box, even though each marble individually is in the box. This is one version of the counting anomaly.

Lewis (1997) maintains that this anomaly shows that spontaneous collapse theories are not viable theories of quantum mechanics. Ghirardi and Bassi (1999), Bassi and Ghirardi (1999, 2001), Frigg (2003), and Parker (2003) argue that spontaneous collapse theories can avoid the counting anomaly, while Clifton and Monton $(1999,2000)$ argue that even though the counting anomaly arises, this is not a problem for spontaneous collapse theories.

Lewis has now switched his position on the viability of the spontaneous collapse theories: he argues (2003a, 2003b) that while spontaneous collapse theories face the counting anomaly, this is not fatal to spontaneous collapse theories. I too have switched my position: while I used to think that counting anomaly is unavoidable, now I think that it is avoidable. This is important, because I now think that were spontaneous collapse theories to face the counting anomaly, they would not be viable theories. (I will argue for this below.)

Whether the counting anomaly arises depends on what link one uses to go from wave function talk to talk of ordinary macroscopic objects. The reason Lewis is not bothered by the counting anomaly is that he sees this link as being purely pragmatic; he thinks that the fundamental ontology of spontaneous collapse theories is that of the wave function. I, on the other hand, believe that this link must have ontological import - as I will argue, understanding spontaneous collapse theories as postulating the existence of only wave functions is not viable. Spontaneous collapse theories must be understood as having ordinary objects (like elementary particles, and objects composed of elementary particles) as part of their fundamental ontology. 
This means that the link must be taken ontologically seriously.

Two different links have been discussed for spontaneous collapse theories: the fuzzy link and the mass density link. For the fuzzy link, I maintain that the counting anomaly does arise. I will argue (pace Lewis) that this shows that the fuzzy link is not a viable interpretative principle. For the mass density link, matters are somewhat more complicated. There are two different versions of the mass density link which are not kept distinct in the literature: what I will call the accessible mass density link and the mass density simpliciter link. For these two links, the counting anomaly does not arise. The accessible mass density link, however, faces other anomalies, and this prevents it from being a viable interpretative principle. The mass density simpliciter link overcomes the problems that the accessible mass density link faces, and provides a viable ontology for spontaneous collapse theories.

\section{Wave Function Ontology}

According to the wave function ontology, the fundamental space in which entities evolve is not three-dimensional, but is instead $3 N$-dimensional, where $N$ is the number of particles standardly though to exist in the three-dimensional universe. The main motivation for this ontology is that the wave function is a $3 N$-dimensional object; the wave function ontology allows one to take the wave function ontologically seriously, as a field evolving in $3 \mathrm{~N}$-dimensional space. According to the wave function ontology, there is no three-dimensional space, at least not 
at the level of fundamental reality. ${ }^{1}$

Clifton and Monton (1999) discuss the view that the wave function ontology is the correct ontology for spontaneous collapse theories. In the context of the fuzzy link, they write:

Fuzzy link semantics, on this view, does not add anything of ontological import to the GRW theory, but simply provides a way of mapping our 'particle' language onto a theory whose fundamental language concerns wavefunctions. (Clifton and Monton 1999, 716) Clifton and Monton are often taken as explicitly endorsing this view, but in fact their position is more nuanced. Their claim is a conditional one: if the fuzzy link can legitimately be construed in accordance with the wave function ontology, then the counting anomaly does not pose a problem for the GRW theory. That said, they do seem inclined toward the antecedent of the conditional.

Lewis (2003a, b) explicitly endorses the wave function ontology for spontaneous collapse theories. For example, he writes:

Spontaneous collapse theories are wavefunction-only theories, in the sense that they attempt to explain the behavior of physical systems in terms of the wavefunction dynamics alone, without postulating any ontological extras... (Lewis 2003a, 168) Lewis takes the fuzzy link and the mass density link to provide "a convenient manner of speaking about the wavefunction."

Monton (2002) has given one set of arguments against the wave function ontology, and while I find these arguments persuasive, I will not repeat them here. Instead I will present a

${ }^{1}$ Some would say that the three-dimensional space exists derivatively; they would say that it supervenes on the $3 \mathrm{~N}$-dimensional space. I find such claims hard to understand; how can one sort of space supervene on another? Regardless of how that debate is settled, at the level of fundamental reality, there is no three-dimensional space according to the wave function ontology. 
different sort of argument against the wave function ontology. ${ }^{2}$ My argument relies on a pragmatic maxim, but it's a maxim that has had much force in the history of science: one should not accept theories which radically revise people's common-sense understandings of the world when there are other, equally acceptable theories which do not entail such extreme revision. While I believe that most readers will find this maxim plausible, I recognize that some will not; those readers can consult Monton 2002 for alternative arguments against the wave function ontology.

The reason the wave function ontology entails a radical revision of our common-sense understanding of the world is that our common-sense understanding holds that the world consists of objects with length, breadth, and depth evolving in a three-dimensional space. According to the wave function ontology, claims that objects exist in three-dimensional space are, strictly speaking, false - at the level of fundamental reality, there is no three-dimensional space according to the wave function ontology, there is only $3 N$-dimensional space. Our common-sense understanding of the world is not simply that the world appears to us to have objects evolving in three-dimensional space; our common-sense understanding is that the world does have objects evolving in three-dimensional space. The wave function ontology may be able to account for the appearances, but it is radically revisionary with respect to how we take things to actually be.

In this respect the wave function ontology is similar to the brain in the vat scenario: in the brain in the vat scenario, we think that the world around us is a certain way, but it turns out that we are radically mistaken about the basic facts regarding the world around us; we are actually all

${ }^{2}$ There are parallels between my argument here against the wave function ontology and the argument of Lewis $(1997,324)$ against the GRW theory. 
brains in vats. In fact, in some ways the wave function ontology is even more radical than the brain in the vat scenario: in the brain in the vat scenario, at least we are correct in thinking that we have brains existing in a three-dimensional space. According to the wave function ontology, even that is incorrect; all that really exists is a wave function field evolving in a $3 \mathrm{~N}$-dimensional space. Just as we think that there is strong prima facie reason to reject the brain in the vat scenario, because of its radically revisionary implications for common-sense ontology, so there is a strong prima facie reason to reject the wave function ontology.

The "prima facie" qualifier is important. I have argued that spontaneous collapse theories with the wave function ontology are radically revisionary with respect to our common-sense understanding of the world. But are there alternatives which are not radically revisionary? If there are not, then arguably the empirical evidence for quantum mechanics forces us to accept a such a radically revisionary theory. But in fact, there are less radically revisionary ontologies. I will show below that spontaneous collapse theories can be interpreted with a more common-sensical ontology than the wave function ontology. Also, there are other versions of quantum mechanics which have more ordinary ontologies: a good example is Bohm's theory. According to the standard interpretation of Bohm's theory, the world consists (at least in part) of point particles evolving in a three-dimensional space. Bohm's theory can be interpreted in terms of the wave function ontology, but Bohm himself was against this:

While our theory can be extended formally in a logically consistent way by introducing the concept of a wave in a $3 \mathrm{~N}$-dimensional space, it is evident that this procedure is not really acceptable in a physical theory... (Bohm 1957, 117)

While Bohm does not say it explicitly, one gathers that the reason it is not acceptable to interpret 
his theory in that way is that such an understanding does not match the world as we experience it. While it is mathematically viable to represent the theory as consisting of objects evolving in $3 \mathrm{~N}$ dimensional space, it is not physically viable, because $3 N$-dimensional space is not an accurate representation of the physical, three-dimensional world.

It follows that the pragmatic maxim cited above leads one to reject spontaneous collapse theories with the wave function ontology in favor of theories which are less revisionary with respect to our common-sense understanding of the world. These less revisionary theories include spontaneous collapse theories which take ontologically seriously the link one uses to go from wave function talk to talk of ordinary macroscopic objects. I will first consider the fuzzy link.

\section{The Fuzzy Link}

The basic idea behind the fuzzy link is that the eigenstate-eigenvalue link, at least for the case of position, is too strict. An object should count as being located in a region as long as most of the object's wave function support is associated with that region. More precisely, for an $n$ particle system, the fuzzy link says:

'Particle $p_{1}$ lies in region $R_{1}$ and ... and $p_{n}$ lies in $R_{n}$ ' iff the proportion of the total squared amplitude of $\psi\left(t, \mathbf{r}_{1}, \ldots, \mathbf{r}_{n}\right)$ that is associated with points in $R_{1} \times \ldots \times R_{n}$ is greater than or equal to $1-\varepsilon$ (where $0.5<\varepsilon<1)$.

While there has been a fair amount of controversy about this point, I maintain that the counting anomaly does arise for spontaneous collapse theories with the fuzzy link ontology. Ghirardi and Bassi (1999) argue that the fuzzy link does not face the counting anomaly, but there seems to be 
widespread agreement that Clifton and Monton (1999) have shown their argument to be mistaken. In their reply to Clifton and Monton, Bassi and Ghirardi (2001) switch the terms of the debate to the mass density link; I will take this up in the next section. Frigg (2003) gives a different argument for the claim that the fuzzy link does not face the counting anomaly, but I endorse Lewis's (2003b) refutation of Frigg's argument. I have nothing to add to Lewis's refutation, so I will not go into the debate here.

Given that the fuzzy link faces the counting anomaly, is this a problem for the fuzzy link? I maintain that it is a problem, and while many others agree with me, there is a controversy about what the problem actually is. I will show that Lewis (1997) incorrectly diagnoses the problem, and I will defend my own answer.

Lewis considers a system of $n$ non-interacting marbles, each in the state $\mid \psi$, discussed above. Let us call the state of such a system state $\mid \Psi$, . Lewis $(1997,318)$ maintains that state $\mid \Psi$, "cannot be one in which all $n$ marbles are in the box, since there is almost no chance that if one looks one will find them all there." Lewis (2003a) elaborates on this claim, in the course of replying to Clifton and Monton's contention that the counting anomaly can never be made manifest. Clifton and Monton argue that a measurement of the number of marbles in the box at time $t$ will result in some number $k \# n$, and at time $t$ it will be the case that there are exactly $k$ marbles in the box. Lewis (2003a, 167) says that this sort of measurement actually "is precisely the means by which [the counting anomaly is] made manifest." He says that the fact that one will most likely get a result $k<n$ shows that not all marbles are in the box, even though "if one measures the position of any individual marble in state $[\mid \psi$,$] , one will almost certainly get the$ result that it is the box." Lewis concludes that the counting anomaly can be made manifest. 
The key to seeing the error in Lewis's reasoning is to note that, as Lewis implicitly admits, it is not certain that a measurement of the position of a marble in state $\mid \psi$, will give the result that the marble is in the box. Instead it is "almost" certain. Even assuming a flawless measuring apparatus, there is a very small probability that the marble will be found outside the box. This probability is the same probability that, when the measurement occurs, the spontaneous collapses will happen in such a way that the marble/measuring apparatus system ends up in a state where most of the wave function support for the marble is associated with the region outside the box. The reason this is the case is that a flawless measuring apparatus will have its pointer perfectly correlated with the position of the marble: whatever the amplitude associated with state |in, of the marble is, that same amplitude will be associated with the state |'in', of the measuring apparatus. Thus, for a marble initially in state $\mid \psi$, , just as there is a $|b|^{2}$ probability of finding the marble outside the box, there is a $|b|^{2}$ probability that the measuring apparatus will record that the marble is outside the box.

Suppose that the measuring apparatus does record that the marble is outside the box. What is the state of the marble/measuring apparatus system in that circumstance? The state of the system is of the form

$$
c \mid \text { in, |'in', }+d \text { |out,|'out', }
$$

where $|c|^{2}+|d|^{2}=1$. If it were the case that $|c| »|d|$, that would be a state where, according to the fuzzy link, the measuring apparatus records that the marble is in the box. But we are supposing that the measuring apparatus records that the marble is outside the box. It follows that the state must be such that $|d| »|c|$. The spontaneous collapses have happened in such a way that the marble is outside the box, and the pointer records that the marble is outside the box. 
Moreover, there is nothing special about the measuring apparatus here - the marble, like the measuring apparatus, is a macroscopic system, and so is subject to frequent spontaneous collapses. Just as the marble/measuring apparatus system can evolve to a state where the marble is outside the box, so can the marble even when it has not interacted with the measuring apparatus.

Now we can demonstrate the incorrectness of Lewis's claim that the state $\mid \Psi$, "cannot be one in which all $n$ marbles are in the box, since there is almost no chance that if one looks one will find them all there." Consider an $n$-marble system which starts out in state $\mid \Psi_{,}$; this is a state in which all $n$ marbles are in the box. At some later time, though, the system might not be in that state; with enough marbles we can expect spontaneous collapses to happen in such a way that some end up outside the box. Thus, when one makes a measurement, one need not find that all the marbles are in the box, even though the system started out in a state where all the marbles were in the box. Supposing one does not find all the marbles in the box, the reason one does not is that some of the marbles are no longer in the box. It is this sort of reasoning that motivates Clifton and Monton's (1999) argument that the counting anomaly can never be made manifest; Lewis has not provided a good refutation of Clifton and Monton's argument.

So why is the counting anomaly a problem for the fuzzy link, given that the anomaly can never be made manifest? The reason is that what the counting anomaly really amounts to is a logical anomaly. On the assumption that the fuzzy link is true, spontaneous collapse theories entail a contradiction. According to the fuzzy link, an $n$-marble system in state $\mid \Psi$, is one where each individual marble is in the box. For sufficiently large $n$, the fuzzy link entails that it's not the case that all $n$ marbles are in the box. Depending on how one looks at it, this is a violation of 
either conjunction introduction or universal generalization: marble 1 is in the box, marble 2 is in the box, ..., marble $n$ is in the box, but it's not the case that (marble 1 is in the box \& ... \& marble $n$ is in the box); it's not the case that all $n$ marbles are in the box.

By my lights, the fact that the fuzzy link entails a contradiction is reason enough to reject it. There are other options, though. One could argue that, since the wave function is all that really exists, the fuzzy link only has limited pragmatic applications, and hence we shouldn't be worried that applying it sometimes leads to contradictions. As I have argued above, though, an appeal to the wave function ontology is inappropriate. One could instead argue that a new logic is needed, call it collapse quantum logic, where a classical logical principle like conjunction introduction or universal generalization is not permissible. Such a move would raise a number of contentious issues regarding the possible a posteriori status of logic and basic principles of belief revision. While I will not go into these issues here, I will simply report my belief that a transition to a deviant logic should be a last resort. Other things equal, an ontology that does not require a revision of logic and does not entail contradictions is to be preferred. The mass density link arguably provides such an ontology.

\section{The Mass Density Link}

The mass density link was proposed by Ghirardi, Grassi, and Benatti (henceforth GGB) in 1995. The basis of the ontology is the mass density function, $m(\mathbf{r}, t)$. To define this mass density function, GGB first introduce particle number density operators, denoted by $N^{(k)}(\mathbf{r})$. Each operator corresponds to the number of particles of type $k$ that exist at point $\mathbf{r}$ of space. Next, mass 
density operators are defined:

$$
M(\mathbf{r})=3_{k} m_{k} N^{(k)}(\mathbf{r}),
$$

where $m_{k}$ is the mass of a particle of type $k$. (Here and elsewhere I suppress the time variable.) Where $\mid \Phi$, is the universal state vector, the mass density function at position $\mathbf{r}$ is defined as

$$
m(\mathbf{r})=\$ \Phi|M(\mathbf{r})| \Phi, .
$$

The mass density simpliciter link holds that the distribution of mass in the universe is governed by $m(\mathbf{r})$. I will defend this link below.

First, though, I will consider an alternative link, the accessible mass density link. According to this link, only accessible mass density is real (where the notion of accessibility will be defined below.) I do not want to claim that anyone has endorsed this link, but Ghirardi and his colleagues sometimes say things which have led people to believe that they endorse this link, as I will discuss below.

To give their criterion for when mass is accessible, GGB start by defining the mass density variance:

$$
v(\mathbf{r})=\$ D\left|[M(\mathbf{r})-\$ \Phi|M(\mathbf{r})| \Phi,]^{2}\right| \Phi, .
$$

They then make the simplifying assumption that space is discrete, and hence replace the functions $m(\mathbf{r})$ and $\vartheta(\mathbf{r})$ with $m_{i}$ and $\vartheta_{i}$ for the $i^{\text {th }}$ cell. The ratio $\vartheta_{i}$ is defined by:

$$
Q_{i}^{2}=v_{i} / m_{i}^{2}
$$

The mass $m_{i}$ in the $i^{\text {th }}$ cell is defined as accessible iff

$$
a_{i} \ll 1 .
$$

Ghirardi and his colleagues sometimes use "objective" as a synonym for "accessible", seemingly 
emphasizing the idea that only accessible mass density is real.

To see some of the consequences of the accessible mass density link, consider the following example. GGB consider an $N$-particle system in the following state, where $\mid \Psi^{\mathrm{A}}$, corresponds the the system being localized in region $\mathrm{A}$ and $\mid \Psi^{\mathrm{B}}$, corresponds to the system being localized in region B, spatially separated from A:

$$
\mid \Psi^{+},=1 / \%\left(\left|/ \Psi^{\mathrm{A}},+\right| \Psi^{\mathrm{B}},\right)
$$

GGB consider sending a test particle between region $\mathrm{A}$ and region $\mathrm{B}$, and point out that such a particle will have inaccessible mass density (since in one branch of the superposition it is gravitationally deflected toward region A, and in the other branch it is deflected toward region B). They write:

nowhere in the universe is there a density corresponding to the density of the test particle. In a sense, if one would insist in giving a meaning to the density function, he would be led to conclude that the particle has been split by the interaction into two pieces of half its density. This analysis shows that great attention should be paid in attributing an 'objective' status to the function $m(\mathbf{r})$. (GGB, 17)

The problem with the accessible mass density link is that it really does have the consequence that the test particle with inaccessible mass is nowhere in the universe: since its mass density is not accessible, it is not real. For a microscopic test particle, its mass could be inaccessible for a long time. In fact, according to the accessible mass density link, objects would often be popping out of and into existence, as the accessibility of their mass changed. While I do not have a knock-down argument as to why this is unacceptable, I maintain that this is a serious anomaly. I admit that the evolution of systems according to quantum mechanics is non-classical, but the regular 
disappearance and reappearance of particles, where sometimes the disappearance is for extended periods of time, moves beyond the realm of the benignly non-classical and into the realm of the anomalous.

One might be tempted to put the concern in terms of conservation of energy: energy is not conserved when the test particle goes out of, and then comes back into, existence. But in fact proponents of spontaneous collapse theories already reject the principle of conservation of energy - energy is not conserved when a GRW collapse happens, for example. The best way to explain the anomaly is just to point out how strange it is to have a physics which entails that objects regularly disappear and reappear. While this is not a fatal blow to spontaneous collapse theories, it would be better if there existed an ontology that did not have such untoward consequences. As I will show, the mass density simpliciter link fulfills this desideratum.

I am not the only person who endorses the mass density simpliciter link. While some people have concluded from passages such as the one above that Ghirardi endorses the accessible mass density link, in fact Ghirardi (personal communication, June 2003) assures me that he endorses the mass density simpliciter link. This is not clear, though, even in Ghirardi's most recent writings. For example, in a long review article, Bassi and Ghirardi (2003) consider the test particle with inaccessible mass density that GGB discuss, and they write:

nowhere in the universe one can "detect" or "perceive" a density corresponding to the density of the test particle. In a sense, if one would insist in giving a meaning to the density function he would be led to conclude that the particle has been split by the interaction into two pieces of half its density. This analysis shows that great attention should be paid in assuming that the function $m(\mathbf{r})$ describes the actual state of affairs. 
(Bassi and Ghirardi 2003, 88)

This first sentence suggests that accessibility is a mark of what is detectable, and this is perfectly compatible with the mass density simpliciter link: it may well be the case that inaccessible mass is not detectable, but that does not mean that it is not real. The last sentence, though, seems to reject the mass density simpliciter link. According to the mass density simpliciter link, m(r) does describe the actual state of affairs. I conclude that, while Ghirardi is a proponent of the mass density simpliciter link, it is not always transparent in his writings.

I will now turn to defending the mass density simpliciter link. Sometimes I will use the term "mass density link"; in these circumstances I am referring to both the accessible mass density link and the mass density simpliciter link. I will present four lines of defense.

(1) I will start by showing that the mass density link is better than the fuzzy link, because the mass density link does not face the counting anomaly. This point is emphasized by Bassi and Ghirardi (1999, 2001), and is partially conceded by Clifton and Monton (2000). The reason the mass density link does not face the counting anomaly is that, for each marble in state $\mid \psi$, almost all the mass of the marble is located inside the box. For an $n$-marble system in state $\mid \Psi$, (where each marble is in state $\mid \psi$, , , it is natural to view this system as one where all $n$ marbles are in the box.

This fact about the distribution of mass is noted by Lewis (1997, 327). Nevertheless, Lewis maintains that the mass density link faces the counting anomaly: he thinks it is illegitimate to claim that all $n$ marbles are in the box, given that if one were to look one almost certainly wouldn't find all of them there. But this is an instance of the erroneous reasoning I discussed in the previous section. There is a high probability that some of the marbles will jump outside the 
box, so just because the marbles are all in the box at a certain time, it does not follow that they will all be in the box when one looks.

(2) Clifton and Monton $(2000,161)$ do not believe that the mass density link is better than the fuzzy link. They pose a trilemma for the mass density link:

either conjunction introduction fails [i.e. the counting anomaly holds], mass talk must be divorced from position talk, or the intuitive connection between either of these kinds of talk and a system's dispositions ... must be severed.

Clifton and Monton (2000, 158) suggest that, if Bassi and Ghirardi accepted the second or third horn of the trilemma, they would be accepting an anomaly that is as "equally surprising" as the counting anomaly. While I used to agree with this claim, I now believe that this is not the case. As discussed above, I believe that the counting anomaly is a serious anomaly, because it generates contradictory claims about fundamental ontology, while Clifton and Monton (1999) seem inclined towards the view that the counting anomaly is not that serious. Assuming that I am right in claiming that the counting anomaly is a serious anomaly, the second and third horns should be preferred, as long as they do not lead to any fundamental problems. I will now show that this is the case.

Clifton and Monton $(2000,160)$ say that one can reach the second horn via the following reasoning:

if one wants to maintain that all the mass of the marbles is objectively in the box, together with the fact that the probability of finding them all there is vanishingly small, then one is committed to a radical breach between mass and location talk.

In fact, this line of reasoning is incorrect. When a marble is found outside the box, the reason is 
that its wave function support is concentrated outside the box, so almost all of its mass is located outside the box. Just because almost all of the mass of each marble is located in the box at some time, it does not follow that the almost all of the mass of each marble is located in the box at some later time when one measures the locations of the marbles.

Bassi and Ghirardi recognize that the second horn of the trilemma holds true for the accessible mass density link, but their reasoning is different from that of Clifton and Monton. Bassi and Ghirardi $(2001,127)$ maintain that the reason the second horn holds is that there are wave function tails:

These tails require a divorce of position talks from mass talks, but ... this divorce is absolutely negligible and experimentally undetectable.

The reason the tails require a divorce of position talk from mass talk is that, when the system is in state $\mid \Psi_{,}$, each marble is in the box, while it's not the case that the amount of mass in the box is $n m$.

Is this divorce of position talk from mass talk a serious anomaly? I think that Bassi and Ghirardi overstate the case: presumably there is, in principle, an experimentally detectable difference between a box with contents of mass $n m$ and a box with contents of mass $|a|^{2} n m$. But I agree with their sentiment that this anomaly is not serious. The claim that a marble is located in a box, while the mass of the marble in the box is not the classically expected $m$, but instead $|a|^{2} m$, is simply not that surprising of a claim, when compared to the other surprising aspects of quantum mechanics. The claim becomes no more surprising when one considers an $n$-marble system.

I will now argue that the third horn of Clifton and Monton's trilemma similarly does not 
lead to any fundamental problems. The third horn says that the intuitive connection between mass talk and a system's dispositions must be severed, and the intuitive connection between position talk and a system's dispositions must be severed. What Clifton and Monton have in mind is the idea that it is strange to say that the mass/position of the system is such that all $n$ marbles are in the box, given that the disposition of the system (revealed by measurement) is for not all the marbles to be in the box. But I have already shown that this way of thinking is erroneous. The disposition of the system is to have some of the marbles jump outside the box, so just because the masses/positions of the marbles are in the box at a certain time, it does not follow that they will all be in the box when one looks.

I conclude that Clifton and Monton's trilemma is not a problem for the mass density link. Endorsing the second and third horns does cause any trouble.

(3) I will now show that the mass density link does not face the tails problem. As I discussed in the first section, the eigenstate-eigenvalue link entails that any particle is spread throughout an unbounded region of space, since wave functions have noncompact support (due to their 'tails'). The accessible mass density link can easily evade the tails problem, because the mass density in the regions of space associated with the wave function tails is inaccessible (GGB, 25), and hence not real. But what about the mass density simpliciter link?

It is true that, according to the mass density simpliciter link, every particle is spread throughout an unbounded region of space. In this sense, the mass density simpliciter link has the same consequence as the eigenstate/eigenvalue link. But the mass density simpliciter link gives more information about the properties of a particle than the eigenstate/eigenvalue link does. Specifically, the mass density simpliciter link specifies what the mass density of a particle is 
throughout space. In most of space, the mass density of a particle is almost zero.

To see whether this is enough to solve the tails problem, we have to think about what the tails problem actually amounts to. The tails problem is simply the problem that there is a prima facie incompatibility between the result one gets from the eigenstate/eigenvalue link, that all objects are spread throughout an unbounded region of space, and the result one gets from everyday observation, that macroscopic objects are highly localized. The mass density simpliciter link resolves this prima facie incompatibility - it can explain why macroscopic objects appear highly localized. The reason macroscopic objects appear highly localized is that most all of their mass is concentrated in a small region of space, the region where the object appears to be localized.

I admit that, for the mass density simpliciter link to solve the tails problem, a certain assumption about psychophysical parallelism needs to be made. But the assumption is a reasonable one. According to the mass density simpliciter link, each of the particles in one's brain is located in an unbounded region of space. If mental states supervened just on particle location, then presumably the appropriate mental states would not supervene on brain states - the evolution of the unbounded regions of space in which the particles are located presumably would not be sufficient for mental states to exist and evolve in the appropriate way. But there is no need to suppose that mental states supervene just on particle location; instead we can suppose that mental states supervene on the distribution of mass. Since the masses of particles in a brain are concentrated in the appropriate regions of space, it is reasonable to assume that the appropriate mental states supervene on those mass concentrations.

I conclude that the mass density simpliciter link solves the tails problem. But this does 
not yet show that the mass density simpliciter link is anomaly-free; there is one final issue to consider.

(4) GGB implicitly present an argument against the mass density simpliciter link; I will call this the test particle problem. Consider the discussion from the previous section of the test particle interacting with a system in state $\mid \Psi^{+}$, . GGB suggest that it's problematic that the mass of the test particle gets split in two, with the two pieces of matter heading in different directions.

Why do GGB imply that this is problematic? This evolution is certainly non-classical, but that's not a reason to object; quantum mechanics itself is non-classical. I take it that GGB are bothered by the fact that the mass density in regions $\mathrm{A}$ and $\mathrm{B}$ are equal, and yet the test particle doesn't behave as if it's passing between two regions of equal mass. GGB write that

The unacceptable features find their origin in the fact that, when the macrostate is $\mid \Psi^{+}$, while the density function takes the value of about $1 / 2 \mathrm{~g} / \mathrm{cm}^{3}$ within regions $\mathrm{A}$ and $\mathrm{B}, \ldots$ if a measurement like process (such as the passage of the test particle in between A and B) occurs, things proceed in such a way that it is incompatible with the above density value. (GGB, 19)

The problem here is that GGB are assuming that the future evolution of a system is determined, at least in part, by the present distribution of mass density. But actually, the future evolution is determined solely by the quantum state of the system. For it to be the case that the future evolution of a system is incompatible with the present mass distribution, one would need to give a dynamics specifying how systems evolve given an initial distribution of mass. It's actually the case that the dynamics of spontaneous collapse theories only depends on the quantum state; the evolution of a quantum state does not depend on the mass density. Instead, mass density is 
epiphenomenal: the mass density at a particular time is determined by the quantum state at that time, but the mass density does not have any influence on the future evolution of the system. Thus, GGB's claim of incompatibility is illegitimate.

I conclude that GGB have not given a good argument for why the mass associated with the test particle is not real; GGB have not given a good argument against the mass density simpliciter link. In fact, all the arguments I have considered against the mass density simpliciter link are unsuccessful. I cannot see any other reason to reject the link, so I conclude that the mass density simpliciter link is free of any serious anomalies.

\section{Conclusion}

The philosophical virtues of spontaneous collapse theories raise the question of whether these theories can be given a viable interpretation. It turns out that a viable interpretation does exist - the mass density simpliciter link, which specifies that the mass density in a region of the universe is proportional to the square of the wave function amplitude in that region. The mass density simpliciter link has the consequence that objects exist in ordinary three-dimensional space; this makes it superior to the wave function ontology. The mass density simpliciter link entails that, in a situation where each of $n$ marbles is in a box, it's the case that all $n$ marbles are in the box; this makes it superior to the fuzzy link. Also, the mass density simpliciter link entails that ordinary objects normally do not go out of and then back into existence; this makes it superior to the accessible mass density link. Moreover, the mass density simpliciter link solves the tails problem and the test particle problem. In sum, the mass density simpliciter link solves 
the problem of ontology for spontaneous collapse theories.

\section{Acknowledgments}

This article is dedicated to the memory of Rob Clifton, who inspired me to think deeply about these issues (and many others). I thank GianCarlo Ghirardi and Daniel Parker for helpful comments. Some of the ideas in this article were first presented in my dissertation; for dissertation assistance I thank Frank Arntzenius, Gordon Belot, Jeff Bub, and Bas van Fraassen. 


\section{References}

Albert, D. (1994). The foundations of thermodynamics and the approach to thermodynamic equilibrium. The British Journal for the Philosophy of Science, 45, 669-677.

Albert, D. (2000). Time and chance. Cambridge, MA: Harvard University Press.

Bassi, A., and Ghirardi, G.C. (1999). More about dynamical reduction and the enumeration principle. The British Journal for the Philosophy of Science, 50, 719-734.

Bassi, A., and Ghirardi, G.C. (2001). Counting marbles: reply to Clifton and Monton. The British Journal for the Philosophy of Science, 52, 125-130.

Bassi, A., and Ghirardi, G.C. (2003). Dynamical reduction models. Physics Reports, forthcoming. Preprint quant-ph/0302164 available at xxx.lanl.gov.

Bohm, D. (1957). Causality and chance in modern physics. London: Routledge.

Clifton, R., and Monton, B. (1999). Losing your marbles in wavefunction collapse theories. The British Journal for the Philosophy of Science, 50, 697-717.

Clifton, R., and Monton, B. (2000). Counting marbles with 'accessible' mass density: a reply to Bassi and Ghirardi. The British Journal for the Philosophy of Science, 51, 155-164.

Frigg, R. (2003). On the property structure of realist collapse interpretations of quantum mechanics and the so-called 'counting anomaly'. International Studies in the Philosophy of Science, forthcoming.

Ghirardi, G.C. and Bassi, A. (1999). Do dynamical reduction models imply that arithmetic does not apply to ordinary macroscopic objects?. The British Journal for the Philosophy of Science, 50, 49-64.

Ghirardi, G.C., Grassi, R., and Benatti, F. (1995). Describing the macroscopic world: closing the circle within the dynamical reduction program. Foundations of Physics, 25, 313-328.

Ghirardi, G.C., Rimini, A., and Weber, T. (1986). Unified dynamics for microscopic and macroscopic systems. Physical Review D, 34, 470-491.

Lewis, P. (1997). Quantum mechanics, orthogonality, and counting. The British Journal for the Philosophy of Science, 48, 313-328.

Lewis, P. (2003a). Counting marbles: a reply to critics. The British Journal for the Philosophy of Science, 54, 165-170. 
Lewis, P. (2003b). Four strategies for dealing with the counting anomaly in spontaneous collapse theories of quantum mechanics. International Studies in the Philosophy of Science, forthcoming.

Monton, B. (1999). Quantum ontology and quantum observers. Princeton University Ph.D. dissertation.

Monton, B. (2002). Wave function ontology. Synthese, 130, 265-277.

Parker, D. (2003). Finding your marbles in wavefunction collapse theories. Studies in History and Philosophy of Modern Physics, forthcoming. 